Sociologie et sociétés

\title{
La géographie du marché de l'art mondial en pleine évolution
}

Cultures des arts régionales et mondialisation culturelle

\section{The changing geography of the global art market}

\section{National leadership, global elites and the diffusion of arts} institutions

\section{Diana Crane}

Volume 47, numéro 2, automne 2015

Trajectoires de consécration et transformations des champs artistiques

Paths to recognition and transformations of artistic fields

URI : https://id.erudit.org/iderudit/1036338ar

DOI : https://doi.org/10.7202/1036338ar

Aller au sommaire du numéro

\section{Éditeur(s)}

Les Presses de l'Université de Montréal

\section{ISSN}

0038-030X (imprimé)

1492-1375 (numérique)

Découvrir la revue

Citer cet article

Crane, D. (2015). La géographie du marché de l'art mondial en pleine évolution : cultures des arts régionales et mondialisation culturelle. Sociologie et sociétés, 47(2), 19-38. https://doi.org/10.7202/1036338ar

\section{Résumé de l'article}

Le présent article examine la participation des artistes asiatiques au système d'art mondial que dominaient naguère les créateurs et les producteurs européens afin de déterminer si l'hégémonie occidentale est en déclin. Parmi les 500 artistes contemporains avec les montants de ventes aux enchères les plus élevés, $49 \%$ étaient d'origine asiatique en 2011-2012, comparativement à $8 \%$ en 2005. La nationalité des membres des comités de sélection des artistes influe énormément sur la sélection des artistes pour les biennales et les foires artistiques. La nationalité des artistes dont les oeuvres sont exposées dans les biennales et vendues aux enchères et dans les galeries dans le cadre de foires artistiques montre qu'il existe une forte " préférence nationale " pour les oeuvres produites au pays ou dans la région. Ainsi, le marché de l'art mondial est segmenté en différentes régions, entre l'Orient et l'Occident, chacune avec ses propres artistes et centres d'art d'importance.
Tous droits réservés @ Les Presses de l’Université de Montréal, 2016
Ce document est protégé par la loi sur le droit d'auteur. L'utilisation des services d'Érudit (y compris la reproduction) est assujettie à sa politique d'utilisation que vous pouvez consulter en ligne.

https://apropos.erudit.org/fr/usagers/politique-dutilisation/ 


\section{La géographie du marché de l'art mondial en pleine évolution}

Cultures des arts régionales et mondialisation culturelle

\section{DIANA CRANE}

Université de Pennsylvanie

13, rue Cassette

75006 Paris

Courriel: craneher@sas.upenn.edu

\section{$\mathrm{D}$} EPUIS LE DÉBUT DU XXI ${ }^{\mathrm{e}}$ SIÈCLE, le marché de l'art contemporain ${ }^{1}$ subit des changements considérables et se développe rapidement. Selon Artprice (2011-2012: 11 ), les ventes aux enchères d'œuvres d'art et leur prix augmentent continuellement. En 2011, 41000 œuvres d'art contemporain ont été vendues aux enchères, un nombre quatre fois plus élevé que les ventes totales en l'an 2000. La valeur de vente de ces œuvres a atteint 915 millions d'euros, soit dix fois plus qu'en l'an 2000.

Cette spectaculaire croissance des prix et des ventes a été causée notamment par le fait que le nombre de milliardaires sur la planète a octuplé de la fin des années 1980 à 2008 (Artprice, 2011-2012: 61; Velthuis et Baia Curoni, 2015: 8). Les gens superriches cherchent à accroître leur prestige en achetant des œuvres d'art très cher. Les goûts et la richesse d'un nombre relativement petit de collectionneurs influent sur ce qui est vendu et à quel prix (Reyburn, 2014a). Même si des pays de toutes les régions de la planète participent à ces événements, la population de nombreux pays ne dispose pas des ressources requises pour développer des marchés d'art locaux ou participer aux

1. L'art contemporain est défini comme les œuvres d'art créées par des artistes nés après 1945 (Artprice, 2011-2012: 11). Voir Heinich (2014) pour une discussion des ambigüités de la définition de l'art contemporain. 
biennales tenues dans d'autres pays (Van Hest, 2012: 139). Dans une certaine mesure, le marché de l'art contemporain peut à juste titre être considéré comme un «club » de pays riches, car pour y participer, il faut un contingent assez imposant de citoyens disposant de ressources suffisantes pour se procurer des œuvres d'art coûteuses dans leur propre pays et ailleurs.

Des études antérieures ont montré qu'une poignée de pays occidentaux dominent le marché de l'art mondial. Quemin (2006: 552; voir aussi Quemin, 2002; Buchholz et Wuggenig, 2005) a conclu que le marché de l'art mondial est composé «d'un nombre réduit de pays occidentaux, dont les États-Unis et l'Allemagne occupent une place prépondérante, et d'une vaste périphérie qui englobe tous les autres États ». Van Hest (2012: 232) arrive à des conclusions similaires en se basant sur une étude des foires d'art, des biennales et des acquisitions des musées de 1970 à 2008. Buchholtz et Wuggenig (2005: 7) ont insisté sur «l'exclusion éhontée de l'Europe de l'Est, de l'Amérique latine, de l'Australie ainsi que de l'Afrique et de l'Asie du centre du... milieu de l'art mondial».

Depuis lors, la Chine a dépassé les États-Unis et l'Europe. Les artistes chinois sont maintenant plus nombreux que les artistes étasuniens et européens sur les listes d'artistes dont les œuvres sont vendues aux prix les plus élevés (Artprice, 2013). Certains pays non occidentaux, notamment en Asie, ont un grand nombre de collectionneurs extrêmement riches, développent des marchés de l'art nationaux et créent des musées et autres institutions des arts, y compris des biennales et des foires artistiques (voir, par exemple, Adeli, 2011). Le fait que cinq pays non occidentaux figurent parmi les dix premiers en 2011-2012 au chapitre des ventes par enchères démontre l'importance croissante de ces pays. La moitié des 40 marchés les plus importants sur la planète sont maintenant en Asie (Artprice, 2013b : 2). Le présent article examine le rôle des participants asiatiques dans le monde de l'art d'avant-garde auparavant dominé par les créateurs et producteurs européens et étasuniens.

L'art contemporain semble avoir une valeur culturelle et symbolique considérable dans les pays non occidentaux. Les institutions artistiques sont des sources importantes de capital culturel pour les villes des pays en développement. Les villes investissent dans des musées et autres institutions culturelles, comme les librairies et les théâtres, dans un effort pour construire un «écosystème» pour les arts (Frazier, 2013; Kong, 2007) qui à son tour attirera les artistes, les entrepreneurs d'arts qui dirigent des galeries et des maisons de vente aux enchères et les touristes. Kong (2007: 384) avance que les villes utilisent trois stratégies pour développer le capital culturel: une stratégie axée sur les producteurs et consommateurs culturels, une stratégie axée sur la production qui encourage la création de produits culturels et une stratégie axée sur les sites qui comporte la création d'une infrastructure culturelle et le développement de propriétés. Elle décrit des exemples de l'utilisation de ces stratégies dans des activités récentes de trois villes d'Asie: Shanghai, Singapore et Hong Kong. Ces stratégies sont des exemples de politiques gouvernementales culturelles qui visent à préserver, à encadrer et à reformuler leurs cultures nationales (Crane, 2002). 
Quelles sont les caractéristiques du marché de l'art contemporain? De manière très générale, le marché de l'art mondial est un réseau gigantesque dans lequel «chaque espace artistique national est imbriqué dans un système mondial de processus culturels et d'échanges» (Moulin, 2000: 81), à savoir, un réseau d'artistes, de marchands, de conservateurs, de directeurs de musée, de maisons de vente aux enchères et de collectionneurs dont les membres participent régulièrement aux foires artistiques et aux biennales et voyagent régulièrement dans les pays qui ont des marchés de l'art établis et des institutions des arts prestigieux. En d'autres mots, le réseau possède ses lieux de rencontre, lesquels facilitent le développement et le maintien du réseau. Leurs activités permettent la diffusion des idées et des tendances et l'avancement de la carrière artistique de quelques élus.

Quemin (2006) a proposé que la configuration de pays du marché mondial suit un modèle centre-périphérie dans lequel certains pays occidentaux dominent et où les pays non occidentaux sont pratiquement invisibles. D'un autre côté, l'importance et la visibilité croissante de l'art non occidental indiquent que le marché de l'art contemporain ne correspond plus à ce modèle (Yogev et Ertug, 2015).

Dans quelle mesure l'hégémonie de l'Occident est-elle en déclin dans le marché de l'art mondial? Les biennales et les foires artistiques exercent-elles une forme d'hégémonie culturelle en sélectionnant les artistes et les galeries de l'Orient et de l'Occident en fonction de leur propre emplacement? Les affiliations nationales des artistes dont les œuvres sont exposées lors des biennales et vendues aux enchères et des galeries participant aux foires artistiques montrent-elles une "préférence nationale», c'est-àdire une préférence pour les œuvres artistiques produites localement ou régionalement?

Des théories de la mondialisation culturelle prédisent que les participants à un marché de l'art mondial fonctionneraient de manière interdépendante. Lechner (2005: 330) parle de «la diffusion planétaire de pratiques, de l'expansion des relations à travers les continents, de l'organisation de la vie sociale à une échelle mondiale et de la croissance d'une conscience mondiale partagée». Buchholtz et Wuggenig (2005: 1) (suivant Giddens, 1990) affirment que les théories de mondialisation insistent sur «l'intégration, les interdépendances réciproques et une conscience commune partagée». Cela mènerait à l'intégration des marchés de l'art nationaux dans « un seul marché mondial unifié et interconnecté ou un champ transnational de l'art contemporain... doté d'une structure institutionnelle et organisationnelle partagée» (Velthuis et Baia Curioni, 2015: 3). Dans quelle mesure le marché de l'art mondial présente-t-il ces caractéristiques?

Dans le présent article, j'examine trois indicateurs de participation nationale au marché de l'art mondial: 1) l'affiliation nationale des artistes sélectionnés pour participer à des biennales dans des villes situées tant en Orient qu'en Occident; 2) l'affiliation nationale de galeries choisies pour participer à d'importantes foires artistiques tant en Orient qu'en Occident; et 3 ) l'affiliation nationale des artistes figurant dans la liste des 500 premiers artistes contemporains au chapitre des ventes aux enchères. Les sélec- 
tions des artistes et des galeries pour les biennales et les foires artistiques reflètent les perceptions et les jugements des conservateurs et des conseillers de ces événements, lesquels sont généralement des personnalités prestigieuses du monde des arts de leur pays. Le palmarès des pays d'origine des artistes qui obtiennent les ventes aux enchères les plus importantes constitue un indicateur objectif de la demande relative sur le marché de l'art mondial pour les œuvres d'artistes de différents pays.

Ces indicateurs ne sont pas exhaustifs. D'autres indicateurs de participation nationale au marché de l'art mondial pourraient être créés en analysant les grandes collections d'art, les échanges entre les principales galeries d'art et le degré auquel les artistes élisent de résider à l'extérieur de leur propre pays dans les villes où les occasions d'accroître leur réputation sont plus nombreuses. Quemin (2002) a examiné la nationalité des artistes dont les œuvres font partie de collections publiques importantes en France et a utilisé la liste des 100 premiers artistes mondiaux présentée dans Kunstkompass (art-compass) et publiée dans Capital, une revue d'affaires allemande (Buchholtz et Wuggenig, 2005). Cette dernière était basée sur les jugements de spécialistes de l'art contemporain, notamment des directeurs de musée et des propriétaires de collections privées importantes.

\section{PARTICIPATION DES ARTISTES OCCIDENTAUX ET ASIATIQUES AUX BIEN NALES}

Les biennales sont des expositions artistiques qui ont lieu tous les deux ans. La première biennale, la Biennale de Venise, a été tenue en 1895 et elle existe toujours. Les biennales sont importantes non seulement en tant que manifestations du marché de l'art mondial, mais aussi pour leurs effets bénéfiques sur le tourisme culturel et la régénération urbaine, même si leur organisation coûte environ six millions d'euros en moyenne (Azimi, 2013a). Le nombre de participants aux biennales est généralement assez élevé. Par exemple, la Biennale de Venise de 2013 a reçu plus de 400000 visites ( «Focus: Biennale de Venise, 55 édition», 2013).

Les biennales jouent un rôle de premier plan dans la mondialisation des institutions d'arts, en attirant des artistes et des galeries provenant de pays de toutes les régions de la planète et en les exposant ainsi à de nouveaux styles et à des modifications de styles existants. Chiu (s.d.) avance que les biennales régionales en Asie ont joué un rôle important dans le développement de l'art contemporain dans la région. Elle dit:

Elles ont favorisé le développement d'une nouvelle identité de l'art contemporain régional... elles ont réuni le travail des artistes de différents pays dans un même lieu et... elles ont créé des espaces dans lesquels les artistes de ces pays pouvaient se rencontrer, présenter mutuellement leurs œuvres et discuter des enjeux et préoccupations communes.

Le nombre de biennales augmente constamment depuis le début des années 1990 (Azimi, 2013a; Morgner, 2014: 277), en partie en raison de la rentabilité interne de ces événements et des bénéfices économiques qu'ils génèrent dans leur contexte urbain. Au début du Xxi ${ }^{\mathrm{e}}$ siècle, il y avait environ 30 biennales (Moulin, 2000: 74). En 2013, la Biennial Foundation (2013) comptait 138 biennales tenues dans 60 pays. Des biennales sont célébrées sur tous les continents. L'Europe et l'Asie ont le plus grand nombre de 
biennales, respectivement 20 et 16. Les États-Unis et le Royaume-Uni ont chacun au moins 10 biennales. La Chine en a 7. L'Afrique, le Moyen-Orient et l'Amérique du Nord (en excluant les États-Unis) ont de 5 à 7 biennales chacun. L'Amérique du Sud et l'Océanie en ont respectivement 3 et 2. Dans la plupart des biennales, les artistes sont invités à présenter leurs œuvres. Dans quelques rares cas, les pays sont invités à choisir un groupe d'artistes dont les œuvres seront présentées ensemble à la biennale.

Van Hest a étudié sept biennales tenues de 1992 à $2007^{2}$. Elle a examiné la représentation d'artistes de différents pays à ces événements dans le but d'évaluer le caractère international réel de ces derniers. Même si 128 pays étaient représentés à sept biennales de 1992 à 2008, Van Hest a conclu que le niveau d'internationalisation de ces événements était limité. Les artistes d'une poignée de pays occidentaux — les États-Unis, le Royaume-Uni, l'Allemagne et la France — avaient de meilleures chances d'être inclus dans ces événements, tout comme les artistes du pays hôte de l'événement et des pays voisins. En d'autres mots, ces événements comportaient des éléments d'hégémonie culturelle occidentale; les influences locales et régionales y ont joué un rôle important.

Pour évaluer le niveau de l'hégémonie occidentale dans la sélection des artistes pour les biennales, nous avons analysé la nationalité des artistes dont les œuvres y ont été récemment exposées. Pour ce faire, nous avons choisi quatre biennales qui ne se spécialisent pas dans un pays ou une région spécifique et qui sont situées dans différentes régions (Europe, Moyen-Orient et Asie): la Biennale de Venise (2013), Documenta (Kassel, Allemagne) (2012) ${ }^{3}$, la Biennale de Sharjah (Émirats arabes unis) (2013) et la Biennale de Gwangju (Corée) (2012). La Biennale de Venise et Documenta sont considérées comme les biennales les plus influentes (Van Hest, 2012: 131). Le nombre de pays représentés à ces biennales varie: Biennale de Venise (36 pays), Documenta (54 pays), Biennale de Sharjah (44 pays) et Biennale de Gwangju (44 pays).

Au total, 16 pays asiatiques étaient représentés à ces quatre biennales. Trois d'entre eux (Chine, Inde et Japon) étaient présents aux quatre biennales et cinq pays asiatiques (Pakistan, Russie, Corée du Sud, Thailande et Vietnam) étaient représentés à trois de ces quatre biennales.

Le Tableau 1 montre qu'en 2013 le niveau de représentation des pays occidentaux et asiatiques a varié considérablement d'une biennale à l'autre. La Biennale de Venise a été la plus occidentale des quatre biennales. Seulement dix pour cent des artistes à la Biennale de Venise 2013 provenaient de l'Asie ou du Moyen-Orient. Documenta, de son côté, avait trois fois plus d'artistes de l'Asie et du Moyen-Orient. Et aux biennales de Sharjah et de Gwangju, les artistes asiatiques et moyen-orientaux dominaient. L'hégémonie occidentale était beaucoup moins forte à ces biennales.

2. Van Hest a étudié les biennales suivantes: Biennale de Gwangju, Biennale de Shanghai, Biennale de Sydney, Biennale d'Istanbul, Biennale de São Paulo, Biennale de Venise et Documenta à Kassel.

3. Documenta n'est techniquement pas une biennale, car elle n'a lieu que tous les cinq ans. 
Tableau 1: Nombre d'artistes de pays occidentaux et asiatiques à quatre biennales, 2012 et 2013 (en pourcentage)

\begin{tabular}{|c|c|c|c|c|}
\hline \multirow[b]{2}{*}{ Pays } & \multicolumn{4}{|c|}{ Biennales } \\
\hline & Venise 2013 & Documenta 2012 & Sharjah 2013 & Gwangju 2012 \\
\hline États-Unis & 25 & 12 & 3 & 9 \\
\hline Allemagne, R.-U., France & 21 & 18 & 8 & 13 \\
\hline Autres Europe ${ }^{a}$ & 35 & 24 & 13 & 9 \\
\hline Total: É.-U., Europe & 81 & 54 & 24 & 31 \\
\hline Chine & 2 & 1 & 5 & 6 \\
\hline Hong Kong & $\circ$ & 1 & o & 1 \\
\hline Autres Asie ${ }^{b}$ & 7 & 21 & 27 & 36 \\
\hline Moyen- Orient ${ }^{c}$ & 1 & 6 & 27 & 9 \\
\hline Total: Asie, Moyen-Orient & 10 & 29 & 59 & 52 \\
\hline Autres pays ${ }^{d}$ & 8 & 18 & 17 & 15 \\
\hline Pays hôte & 8 & 11 & 3 & 22 \\
\hline $\mathrm{N}$ & $(161)$ & (199) & (114) & $(106)$ \\
\hline
\end{tabular}

a Autres pays européens: Albanie, Autriche, Belgique, Bulgarie, Croatie, Chypre, Danemark, Égypte, Hongrie, Irlande, Italie, Pays-Bas, Irlande du Nord, Pologne, Roumanie, Russie, Écosse, Slovaquie, Espagne, Suisse, Ukraine et Yougoslavie.

b Autres pays asiatiques: Afghanistan, Bangladesh, Cambodge, Chine, Géorgie, Inde, Indonésie, Japon, Kazakhstan, Kirghizstan, Corée, Pakistan, Philippines, Russie, Singapore, Corée du Sud, Taiwan, Thaïlande, Ouzbékistan et Vietnam.

c Moyen-Orient: Bahreïn, Égypte, Iran, Iraq, Liban, Palestine, Qatar, Arabie saoudite, Sharjah, Syrie, Turquie et Émirats arabes unis.

¿ Autres pays: Algérie, Argentine, Australie, Brésil, Cameroun, Canada, Chili, Cuba, Éthiopie, Finlande, Ghana, Guatemala, Lituanie, Mexique, Maroc, Nigeria, Norvège, Paraguay, Pérou, Sénégal, Afrique du Sud, Soudan, Tunisie, Venezuela, Vietnam, Zambie et Zimbabwe.

Une comparaison des statistiques produites par Van Hest (2012: 157) et celles des mêmes biennales en 1992 et 2007 montre que la proportion des artistes asiatiques à la Biennale de Venise est demeurée stable à $9 \%$ alors qu'elle a augmenté à Documenta, passant de $7 \%$ à $23 \%$.

La nationalité des membres du comité qui choisit les artistes constitue un facteur important de la sélection des artistes. La Biennale de Gwangju a été l'exception à ce titre parce que «les artistes et les œuvres ont été choisis grâce à la collaboration sans précédent de six directeurs/conservateurs alors que la règle habituelle est qu'un seul ou deux directeurs/conservateurs les choisissent» («Gwangju Biennale 2012: curatorial genius or chaos?» 2012). Ces directeurs artistiques provenaient de six pays de l'Asie et du Moyen-Orient: Chine, Inde, Indonésie, Japon, Corée du Sud et Qatar.

En revanche, les artistes de la Biennale de Venise ont été choisis par un seul conservateur italien qui a remercié six collaborateurs pour leurs conseils, dont aucun n'était non occidental (si l'on se fie à leurs noms) (Gioni, 2013). Le directeur artistique de 
Documenta était italien ( Carolyn Christov-Bakargiev», 2012). Parmi ses onze conseillers, deux n'étaient ni européens ni étasuniens; un d'entre eux était mexicain et l'autre, sud-africain (dOCUMENTA (13) Advisors, 2012). La Biennale de Sharjah a aussi été conservée par une seule conservatrice du Japon, spécialiste de la théorie de l'art, qui a également conservé de nombreuses expositions internationales, y compris plusieurs autres biennales (Yuko Hasegawa, 2013) ${ }^{4}$.

Ces conclusions montrent que la représentation des artistes asiatiques à ces biennales dépend de la participation d'Asiatiques dans le processus de sélection; c'est un exemple frappant de "préférence nationale».

\section{PARTICIPATION DES GALERIES OCCIDENTALES ET ASIATIQUES AUX FOIRES ARTISTIQUES}

Les foires artistiques sont un élément important du marché de l'art mondial (Quemin, 2013; Morgner, 2014). Ce sont des événements à la fois transnationaux et urbains qui réunissent des galeries et des collectionneurs d'œuvres d'art durant une courte période de temps dans une même ville, pour voir une multitude d'œuvres d'art provenant d'un grand nombre de pays. Les galeries ont tendance à générer au moins un tiers de leurs recettes à l'occasion des foires artistiques (Bowley, 2013).

Bien que les premières foires artistiques aient vu le jour dans les années 1960, leur nombre a beaucoup augmenté au cours des deux dernières décennies, passant de cinquante dans les années 1990 à environ 180 en 2013 (Bowley, 2013) et à presque 300 en 2014 (Reyburn, 2014b). Certaines foires artistiques se spécialisent dans l'art d'une région spécifique. Quelques grandes métropoles ont plusieurs foires artistiques. Les foires les plus populaires attirent de 60000 à 150000 visiteurs.

La foire artistique la plus prestigieuse est Art Basel, laquelle a lieu annuellement dans la ville de Bâle, Suisse. Van Hest (2012: 191-192) a examiné la nationalité des galeries qui ont participé à cette foire de 2000 à 2008. Elle a observé que les galeries provenant d'un nombre réduit de pays occidentaux - États-Unis, Allemagne, Suisse, France et Royaume-Uni - étaient les plus nombreuses à cet événement. Tout comme pour les biennales, les galeries du pays hôte, la Suisse dans ce cas-ci, sont surreprésentées. Parmi les quarante pays qui ont participé à cet événement durant cette période, il n'y avait que cinq pays asiatiques, soit la Chine, l'Inde, le Japon, la Corée du Sud et la Russie, et trois pays du Moyen-Orient, soit Israël, le Liban et la Turquie. Seulement un nombre réduit de galeries provenait de chacun de ces pays. Baia Curioni et coll. (2015: 65), qui ont aussi étudié Art Basel, ont trouvé que le pourcentage d'artistes asiatiques en 2005 et 2012 était de 7,2 et de 11,6 respectivement. Les pourcentages de galeries d'art asiatiques étaient de 3,1 et de 5,6 pour cent, respectivement en 2005 et 2011.

4. Le but d'Hasegawa pour la Biennale était de réévaluer l'occidentalocentrisme de la connaissance à l'époque contemporaine et de réexaminer la relation entre le monde arabe, l'Extrême-Orient, l'Afrique du Nord et l'Amérique latine (www.sharjah.org/biennial/sharjah-biennial-1 (consulté le 29/11/2014). 
L'apparition de grandes foires artistiques en Asie montre que l'art asiatique a connu une importante croissance depuis 2006: Beijing (2006), Shanghai (2007), Art Hong Kong (2008) $)^{5}$ et Art Stage Singapore (2011) (Bergman, 2013).

Pour évaluer la participation actuelle des galeries et des foires artistiques asiatiques, nous avons examiné la participation des galeries nationales aux foires artistiques suivantes: Art Basel 2013, Frieze 2013, Art Dubaï 2013, Art Basel Hong Kong 2013, et Art Stage Singapore (2013). Basée à Londres, la foire artistique de Frieze, est considérée comme l'une des foires les plus importantes. Art Dubaï a été choisie parce qu'elle est la première foire artistique au Moyen-Orient et est vue comme «le lieu de rencontre essentiel pour les collectionneurs, les artistes et les professionnels de l'art de partout au Moyen-Orient, en Afrique du Nord, en Asie du Sud et au-delà» (Kendzulak, 2013). Hong Kong est récemment devenu le centre des ventes d'art asiatique en raison de ses avantages géographiques, économiques et fiscaux qui attirent un grand nombre de collectionneurs étrangers et chinois (Artprice, 2011-2012: 36).

Les galeries d'Europe et des États-Unis dominaient les deux foires artistiques occidentales, Art Basel et Frieze (voir le Tableau 2). Quatre-vingt-cinq pour cent des galeries à Art Basel et soixante-dix-neuf pour cent des galeries à Frieze étaient de New York ou d'une ville européenne. La représentation des galeries d'Asie et du Moyen-Orient était minime. Dans les foires non occidentales, la représentation des galeries de New York et des villes européennes était beaucoup plus basse: $53 \%$ à Art Dubaï, $43 \%$ à Art Basel Hong Kong et $27 \%$ à Art Stage Singapore. Les galeries du Moyen-Orient étaient les plus nombreuses à Art Dubaï: $28 \%$. La proportion de galeries asiatiques et moyenorientales était la plus élevée à Art Basel Hong Kong (49\%) et Art Stage Singapore (61\%). Comme dans le cas des biennales, les pays hôtes étaient bien représentés par leurs propres galeries.

L'hégémonie occidentale est très frappante dans les deux foires artistiques occidentales et étonnamment marquée à Art Dubaï. Encore une fois, la nationalité des membres des comités de sélection ou des conseils consultatifs a influé sur celle des galeries sélectionnées. Le comité de sélection d’Art Basel 2013 n'avait aucun membre d'un pays non occidental. La moitié des membres du comité de sélection d'Art Dubaï provenaient de pays non occidentaux tout comme cinq des huit membres du comité de sélection d'Art Basel Hong Kong ( «Art Basel Selection Committee for Hong Kong, », 2013). Les huit membres du comité de sélection d'Art Stage Singapore étaient originaires de pays non occidentaux («For Exhibitors/Art Stage Singapore», 2013)

Ces données montrent que le processus de sélection des foires artistiques ressemble à celui des biennales. La proportion d'artistes asiatiques dépend de la participation d'Asiatiques aux comités de sélection.

5. Art Basel Hong Kong s'appelait Art HK à ses débuts en 2008. Cette foire a été achetée par Art Basel en 2011 (Chen, 2013).

6. Aucune information sur le comité de sélection de la foire artistique de Frieze n'était disponible. 
Tableau 2: Présence des galeries de pays occidentaux et asiatiques à cinq foires artistiques, 2013 (en pourcentage)

\begin{tabular}{|c|c|c|c|c|c|}
\hline \multirow[b]{2}{*}{ Nationalité des galeries ${ }^{a}$} & \multicolumn{5}{|c|}{ Foires artistiques* } \\
\hline & Art Basel & Frieze & Dubaï & Art Basel Hong Kong & Art Stage Singapore \\
\hline New York & 24 & 13 & 7 & 10 & 2 \\
\hline Berlin, Londres, Paris & 31 & 38 & 28 & 15 & 14 \\
\hline Autres villes d'Europe ${ }^{b}$ & 31 & 28 & 18 & 18 & 11 \\
\hline Total: New York, Europe & 85 & 79 & 53 & 43 & 27 \\
\hline Beijing, Shanghai & 2 & 1 & 1 & 9 & 10 \\
\hline Hong Kong & o & o & o & 11 & 5 \\
\hline Autres villes d'Asie ${ }^{c}$ & 4 & 4 & 7 & 27 & 30 \\
\hline Villes du Moyen-Orient ${ }^{d}$ & 2 & 4 & 28 & 3 & 2 \\
\hline Total: Asie, Moyen-Orient & 8 & 9 & 36 & 49 & 61 \\
\hline Autres villes ${ }^{e}$ & 7 & 11 & 11 & 9 & 12 \\
\hline Pays hôte & 6 & 25 & 14 & 11 & 13 \\
\hline $\mathrm{N}$ & (234) & (152) & (75) & (244) & (128) \\
\hline
\end{tabular}

a Lorsqu'une galerie a indiqué une affiliation à deux pays ou plus, nous avons assigné comme pays d'affiliation le premier pays indiqué dans la liste en présumant qu'il était le plus important..

b Autres villes européennes: Amsterdam, Athènes, Bâle, Bruxelles, Cologne, Dublin, Düsseldorf, Exeter, Glasgow, Genève, Graz, Hambourg, Cracovie, Lisbonne, Luxembourg, Madrid, Milan, Naples, Saint-Pétersbourg, Salzburg, Saint-Moritz, Turin, Vérone, Vienne, Varsovie, Zurich ainsi que Bergen, Copenhague, Oslo et Stockholm (Scandinavie).

c Autres villes asiatiques: Bangalore, Chengdu, Jakarta, Kyoto, Manille, Mumbai, Nouvelle Delhi, Osaka, Semarang, Séoul, Singapore, Taipei, Tokyo et Adélaïde, Melbourne, Sydney (Océanie).

d Les villes du Moyen-Orient sont Beyrouth, Dubaï, Istanbul, Jiddah, Manama et Tel- Aviv.

e Autres villes: Adélaïde, Amsterdam, Beverly Hills, Bogota, Buenos Aires, Le Cap, Chicago, Dakar, Douala, Johannesburg, Los Angeles, ville de Mexico, Porto Alegre, Lagos, Reykjavik, Rio de Janeiro, Salisbury, Santiago, San Francisco, São Paulo, Ségou, Tunis et Vancouver

Le prestige des foires artistiques asiatiques a aussi augmenté. En se basant sur des données de 2008, Quemin (2013: 171) affirme:

Aucune foire artistique tenue à l'extérieur d'une poignée de pays de l'Europe de l'Ouest et des États-Unis n'a une influence internationale majeure et n'est en mesure d'attirer les galeries d'art contemporain les plus prestigieuses.

Cependant, à Art Basel Hong Kong en 2013, un nombre considérable de grandes galeries occidentales était présent (Sagot-Duvauroux, 2014). Nous avons identifié les grandes galeries à l'aide de la liste Power 100 de 2013, laquelle est décrite comme «Une liste ordonnée des entités les plus puissantes du monde de l'art contemporain ». Cette liste incluait 24 galeries situées dans des pays occidentaux, dont 16 (67\%) étaient présents à Art Basel Hong Kong. Certaines de ces galeries avaient ouvert une succursale à Hong Kong. Parmi les 24 galeries étasuniennes présentes à la foire de Hong Kong, 6 étaient des galeries figurant dans la liste Power 100, dont la galerie Gagosian et la 
galerie David Zwirner, deux des galeries les plus rentables du monde (Frazier, 2013). La présence de ces galeries est sans aucun doute liée au fait qu'Art Basel s'était associée à la foire artistique de Hong Kong, mais si Art Basel a cherché à avoir pignon sur rue à Hong Kong, c'est parce que sa perception du marché de l'art asiatique a beaucoup changé. La présence des grandes galeries occidentales étaie beaucoup moins évidente à Art Dubaï et à Art Stage Singapore: trois et deux galeries respectivement.

\section{DIFFÉRENCES NATIONALES DANS LES VENTES AUX ENCHÈRES}

Depuis le début de la dernière décennie, le marché aux enchères mondial s'est étendu et diversifié, notamment en Asie. En 2001, Londres et New York dominaient le marché aux enchères avec $80 \%$ des ventes (Artprice, 2013b). En 2011, 45\% des profits des enchères ont été générés en Asie. Beijing et Hong Kong sont maintenant les marchés aux enchères les plus importants de la planète après New York et Londres (Bergman, 2013). Selon Artprice (2013b) ; «Les artistes asiatiques ont généré 35\% des ventes aux enchères et $45 \%$ des profits en 2011/2012.» Treize des vingt premiers marchés de l'art du monde, ou 40,4\%, sont situés en Asie ou au Moyen-Orient (voir le Tableau 3).

Tableau 3: Les 20 premières villes pour la vente aux enchères d'œuvres d'art contemporain (2011 et 2012) ${ }^{a}$

\begin{tabular}{|c|c|c|c|}
\hline \multicolumn{4}{|c|}{ Pourcentage des ventes mondiales d'œuvres d'art contemporain } \\
\hline \multicolumn{2}{|c|}{ Europe/Amériques } & \multicolumn{2}{|c|}{ Asie/Moyen-Orient } \\
\hline New York & 28,1 & Beijing* & 17,8 \\
\hline Londres & 22,5 & Hong Kong* & 14,2 \\
\hline Paris & 2,0 & Shanghai* & 2,4 \\
\hline Stockholm & 0,5 & Hangzhou* & 1,1 \\
\hline Amsterdam & 0,4 & Taipei & 0,9 \\
\hline Vienne & 0,3 & Istanbul & 0,7 \\
\hline Madrid & 0,2 & Dubaï & 0,6 \\
\hline TOTAL & 54,0 & Guangzhou* & 0,6 \\
\hline $\mathrm{N}$ & (7) & & \\
\hline & & Singapore & 0,6 \\
\hline & & Nanjing* & 0,5 \\
\hline & & Séoul & 0,4 \\
\hline & & Tokyo & 0,4 \\
\hline & & Jinan* & 0,2 \\
\hline & & TOTAL & 40,4 \\
\hline & & $\mathrm{N}$ & (13) \\
\hline
\end{tabular}

a

Source: Artprice.com, 2012

$*$ Villes chinoises. 
Les statistiques sur les ventes aux enchères d'œuvres d'art constituent un troisième indicateur de l'importance de la relation des pays occidentaux et asiatiques dans le marché de l'art mondial. Les 500 premiers artistes du marché de l'art mondial ont été classés selon les chiffres de ventes aux enchères de leurs œuvres. Les variations du nombre d'artistes par pays sur la liste indiquent l'importance relative des artistes occidentaux par rapport aux artistes asiatiques.

En 2005, seulement 40 artistes asiatiques figuraient sur la liste des 500 ( «The art market in 2012-The Emergence of Asia,» 2012: 3). Mais en 2011-2012, $49 \%$ des 500 artistes contemporains avec les ventes aux enchères les plus élevées étaient asiatiques (voir Tableau 4). Les artistes chinois étaient beaucoup plus nombreux que les artistes européens et étasuniens: Chine (210), États-Unis (97), Royaume-Uni (33), Allemagne (29) et France (6). La liste comprenait également des artistes des pays asiatiques suivants: Inde (7), Indonésie (5), Japon (10), Corée (6) et Taiwan (5). Parmi les cinquante premiers artistes de la liste, $40 \%$ étaient de Chine, comparativement à $20 \%$ des États-Unis et $10 \%$ du Royaume-Uni, de l'Allemagne et de la France.

\section{Tableau 4: Proportion d'artistes de pays occidentaux et asiatiques parmi les 500 premiers artistes contemporains au chapitre des ventes aux enchères $(2012 / 2013)^{a}$}

\begin{tabular}{|l|c|}
\hline \multicolumn{2}{|l|}{ Pourcentages parmi les 500 premiers artistes contemporains } \\
\hline \multicolumn{2}{|l|}{ Nationalité des artistes } \\
\hline Occident & 19 \\
\hline États-Unis & 14 \\
\hline Allemagne, R.-U., France & 7 \\
\hline Autres pays européens ${ }^{\mathrm{b}}$ & 40 \\
\hline Total: & \\
\hline Asie & 42 \\
\hline Chine & 7 \\
\hline Autres pays asiatiques & \\
\hline Total: & 49 \\
\hline Moyen-Orient ${ }^{d}$ & 4 \\
\hline Autres payse & 7 \\
\hline N & $(500)$ \\
\hline
\end{tabular}

\footnotetext{
a Basé sur «Top 500 contemporary artists (2012/2013): Auction sales from 1st July, 2012 to 30 th June, 2013» (Artprice, 2013).

b $Y$ compris Autriche, Italie, Luxembourg, Pologne, Roumanie, Espagne, Suède et Yougoslavie.

c $Y$ compris Inde, Indonésie, Japon, Corée, Taiwan, et Nouvelle-Zélande.

d $Y$ compris Égypte, Israël, Liban, Arabie saoudite, Syrie et Turquie.

e Y compris Argentine, Australie, Brésil, Colombie, Cuba, Kenya, Mexico, Uruguay et Venezuela.
} 


\section{LE CAS DE LA CHINE}

En moins d'une décennie, la Chine est devenue un des pays les plus importants du marché de l'art mondial. Il représente $90 \%$ du marché de l'art asiatique (Artprice, 2013: 12, 13) et $41 \%$ du marché de l'art mondial. Bien que quelques artistes chinois expatriés aient commencé à vendre leurs œuvres à des collectionneurs et à des musées occidentaux au cours des années 1990, les maisons de ventes aux enchères occidentales n'ont commencé à s'intéresser sérieusement à l'art contemporain chinois en Chine qu'en 2004 (Lau, 2013). Après que la Chine est devenue membre de l'OMC, le gouvernement chinois a permis aux maisons de vente aux enchères étrangères de s'établir à Hong Kong en 2006 ( «Chinese Contemporary Art Today», 2009) même si les maisons de ventes aux enchères internationales ont dû attendre jusqu'en 2013 pour avoir le droit de fonctionner de manière indépendante en Chine continentale (Chen, 2013; Beuve-Méry, 2013). En 2005, après des ventes aux enchères spectaculaires d'œuvres d'artistes chinois à Beijing et Hong Kong, la Chine a pris le troisième rang au chapitre des ventes dans le marché des œuvres d'art contemporain (Artprice, 2013: 12). Par la suite, le prestige de l'art contemporain en Chine a augmenté considérablement en raison de son attrait pour les nouveaux collectionneurs chinois très fortunés (Artprice, 2013: 7).

La montée de la Chine comme un marché d'art a été favorisée par le nombre croissant de milliardaires collectionneurs ${ }^{7}$ et leur intérêt pour l'art comme investissement, comme l'atteste l'expansion des fonds d'investissement chinois spécialisés dans les arts (Artprice, 2013: 12). Les fonds d'investissement contribuent à l'augmentation et à la spirale des prix des œuvres de jeunes artistes (Artprice 2013: 39). En d'autres mots, l'expansion du marché de l'art chinois s'explique principalement en raison de la croissance économique de la Chine. Les collectionneurs chinois achètent presque exclusivement des œuvres d'artistes chinois (Artprice, 2012:14). Ils font preuve d'une forte demande pour les nouveaux artistes, notamment les jeunes artistes, un facteur absent dans d'autres régions (Artprice, 2012:3) qui est à l'origine de l'augmentation des prix des œuvres d'art des jeunes artistes.

En 2002, la part chinoise des ventes aux enchères mondiales était minime (moins de 2\%) (Artprice, 2013: 13). En 2012, cette part avait atteint 41\% (Artprice, 2012: 7 ) alors que la part des trois principaux pays du marché de l'art occidental, les États-Unis, le Royaume-Uni et l'Allemagne, était de $47 \%{ }^{8}$. Avant un ralentissement en 2012, la Chine était le plus grand marché de ventes aux enchères au monde en 2010 et $2011^{9}$. Six maisons aux enchères chinoises figuraient parmi les dix premières maisons de ventes aux enchères au monde durant l'exercice de 2011 (Artprice, 2011-2012: 13).

7. Des musées français ont récemment sollicité des dons de collectionneurs milliardaires chinois (Azimi, 2013c).

8. États-Unis $=27 \%$; Royaume-Uni $=18 \%$; et Allemagne $=2 \%$.

9. Les ventes aux enchères ont diminué de presque $50 \%$ en Chine en 2012. Les marchands et analystes d'art accusent la répression gouvernementale contre l'importation et l'exportation d'œuvres d'art, ainsi que des préoccupations croissantes concernant les faux (Barboza et Bowley, 2013). 
Malgré sa croissance spectaculaire, le marché de l'art chinois comprend plusieurs aspects négatifs. La fraude est généralisée bien qu'elle soit un problème plus sérieux dans le secteur des œuvres d'art traditionnel que dans celui de l'art contemporain (Barboza et Cox, 2013). La première maison de ventes aux enchères chinoise, Poly Auctions, appartient à l'Armée populaire de libération (Bowley et Barboza, 2013). Elle serait un site de blanchiment d'argent et de stratagèmes de pots-de-vin qui contribuent aux prix faussement et artificiellement gonflés des œuvres d'art (Frazier, 2013). Les prix de catalogue indiqués ne sont pas nécessairement les prix que les collectionneurs ont payés. Un autre problème est que les acheteurs omettent de payer les œuvres qu'ils achètent aux enchères (Artprice, 2013: 14).

Le gouvernement chinois appuie les institutions d'arts publics et en même temps il se méfie des institutions privées. Le gouvernement a construit des centaines de musées, y compris 175 musées d'art (Azimi et de Changy, 2013b), mais seulement 450 musées sur 3400 sont privés (Platt, 2012).

L'ambivalence du gouvernement chinois à l'égard des effets politiques potentiels que pourrait avoir l'art contemporain constitue un problème plus sérieux pour cette discipline. Ce problème a surgi à la dernière Biennale de Venise (Platt, 2013). D’un côté, le ministère de la Culture de Chine a appuyé le pavillon de la Chine à la Biennale en raison du prestige de l'événement. D'un autre côté, l'accès au site Web de la Biennale a été bloqué en Chine à cause de messages politiques que contenaient certaines des œuvres d'art. Le gouvernement chinois a arrêté à plusieurs reprises l'artiste contemporain le plus renommé du pays, Ai Weiwei, parce que son travail critique le système politique chinois.

Dans les faits, le marché de l'art chinois est composé de deux cultures artistiques: une culture gouvernementale officielle et une culture de l'art contemporain créée par des artistes d'avant-garde (Platt, 2012). Les musées officiels de l'État refusent d'exposer l'art contemporain, lequel est présenté dans des galeries et des musées privés. Ces derniers représentent une petite proportion (13\%) des 3400 musées que compte le pays.

\section{LE MOYEN-ORIENT: UNE RÉGION ÉMERGENTE}

Des marchés de l'art apparaissent également au Moyen-Orient (Kräussl, 2015), notamment à Doha (Qatar) et à Abu Dhabi et Dubaï (Émirats arabes unis). En ce moment, comme nous l'avons vu, ces artistes sont très peu représentés aux biennales et aux foires artistiques à l'extérieur de la région (voir les Tableaux 1 et 2). Seulement $4 \%$ des 500 premiers artistes contemporains proviennent de pays du Moyen-Orient.

Toutefois, Doha est une capitale d'art à cause de ses investissements dans les musées et ses achats d'œuvres d'art étrangères (Artprice, 2013: 21). Depuis 2008, la ville a ouvert deux nouveaux musées et prévoit en ouvrir une vingtaine de plus. Le budget annuel du gouvernement pour l'acquisition d'œuvres d'art est de presque un milliard d'euros. Doha est aujourd'hui l'acheteur d'art moderne et contemporain le plus important de la planète (Burns et Burns, 2011), alors qu'elle a récemment payé des prix énormes pour des œuvres modernes et de l'après-guerre, notamment des 
peintures de Cézanne ${ }^{10}$, de Rothko, de Francis Bacon et de plusieurs artistes pop ainsi que des œuvres d'artistes contemporains comme Damien Hirst et Takashi Murakami.

La directrice de la Qatar Museums Authority ${ }^{11}$ a déclaré: «Nous nous réexaminons à travers nos institutions culturelles et notre développement culturel. L'art devient une partie très importante de notre identité nationale» (Pogrebin, 2013). La revue Art Review l'a nommée personnalité la plus puissante du monde des arts en 2013, à la tête d'une liste de 100 artistes, marchands d'art, collectionneurs, conservateurs et directeurs d'institutions artistiques prestigieuses de l'Occident (Power 100, 2013). Seulement $16 \%$ des noms figurant sur la liste étaient des personnes du Moyen-Orient ou de l'Asie.

Abu Dhabi, Émirats arabes unis, est un autre centre d'art de la région. Elle aussi investit massivement dans les musées, y compris quatre musées conçus par des architectes occidentaux de renom (Artprice, 2013: 23). Ses musées ont des budgets d'acquisition énormes et ils achètent les œuvres d'artistes du Moyen-Orient et asiatiques ainsi que d'artistes étasuniens et européens. Dubaï, qui est l'hôte d'une foire artistique importante dans la région, figurait parmi les 20 premières villes de la planète au chapitre des ventes aux enchères en 2011-2012 (voir le Tableau 3). Elle profite de la turbulence politique au Moyen-Orient. Les artistes, les galeries et les collectionneurs d'Égypte, d'Iran, du Pakistan, du Soudan, de la Syrie et de Tunisie s'y sont récemment établis (Hamdon, 2013).

\section{LE MARCHÉ DE L'ART MONDIAL: SEGMENTÉ OU INTÉGRÉ ?}

Les conclusions de cette étude indiquent que quelques pays occidentaux, principalement les États-Unis, ne dominent plus le marché de l'art mondial. Quelles sont les caractéristiques du marché de l'art mondial aujourd'hui? Dans quelle mesure les diverses régions du marché de l'art mondial fonctionnent-elles de manière interdépendante comme semblent l'indiquer les théories de la mondialisation culturelle?

Peut-être que les diverses régions du marché de l'art mondial sont des communautés distinctes qui interagissent minimalement les unes avec les autres. En d'autres mots, le marché de l'art mondial pourrait être segmenté avec un peu de chevauchement dans les activités des diverses régions. Ou au contraire, l'on pourrait s'attendre à ce que les participants de certaines ou de toutes les régions achètent ou vendent des œuvres d'art dans d'autres régions qui seraient intégrées dans un réseau mondial.

Comme nous l'avons vu, les biennales et les foires artistiques, notamment en Occident, ont tendance à sélectionner des artistes et des galeries de leur propre région. Elles ont tardé à reconnaître les changements qui surviennent dans d'autres parties de la planète. Leurs gardiens continuent d'exercer une sorte d'hégémonie culturelle dans la sélection des artistes et des galeries pour ces événements.

10. La Qatar Museums Authority a déboursé 250 millions de dollars pour la peinture de Cézanne, le prix connu le plus élevé de l'histoire pour une peinture (Pogrebin, 2013).

11. Sheikha Al-Mayassa bint Hamad bin Khalifa Al-Thani (Brown, 2013). 
Le comportement des collectionneurs d'art est un autre signe d'un marché de l'art mondial segmenté. Achètent-ils essentiellement des œuvres d'art de leur propre région? On ne peut répondre à cette question qu'indirectement, car il n'existe pas d'études adéquates sur les collectionneurs d'art.

En 2013, une liste des dix premiers artistes contemporains en Europe, en termes de ventes aux enchères, n'incluait qu'un seul artiste asiatique (Artprice, 2013: 27). La liste des dix premiers artistes contemporains aux États-Unis, en termes de ventes aux enchères, incluait deux artistes asiatiques (Artprice, 2013: 11). La même liste d'artistes contemporains en Chine ne comprenait aucun artiste occidental (Artprice, 2013: 13). Selon Artprice (2013: 15), la majorité des acheteurs chinois n'achètent que des ouvres locales. La majorité des artistes chinois les plus chers ne sont même pas connus des collectionneurs occidentaux (Artprice, 2011/2012: 24) parce que leurs œuvres ne correspondent pas aux goûts occidentaux. Ces œuvres sont souvent subventionnées par le gouvernement chinois et approuvées par les censeurs.

Ces données semblent indiquer que le marché de l'art mondial est segmenté par régions, Orient et Occident. Chaque région a ses propres grands artistes et grands centres d'art. Aujourd'hui, des artistes chinois dominent le segment asiatique du marché de l'art mondial. Beijing, Hong Kong et Singapore en sont les grands centres. New York domine le segment occidental du marché de l'art mondial ${ }^{12}$, suivie de Londres et de Berlin. Par conséquent, on ne peut plus affirmer que quelques pays européens et les États-Unis dominent l'ensemble du marché de l'art mondial.

Il se peut que cette segmentation du marché de l'art mondial soit temporaire. La Chine était la région vedette à l'Armory Fair de New York en 2013 ( «China focus for next Armory fair», 2013). Le conservateur de l'exposition y a affirmé qu'une grande partie des œuvres d'art produites en Chine aujourd'hui est «injustement rejetée par le public occidental».

\section{CONCLUSION}

La présente étude contribue à la compréhension de la mondialisation culturelle. Elle montre que cette dernière prend la forme de cultures régionales dans le marché de l'art mondial. Le marché de l'art mondial ne suit plus un modèle de centre-périphérie dans lequel les pays occidentaux dominent le marché et les pays non occidentaux sont relégués à la périphérie. À la place, le marché de l'art mondial est segmenté par régions qui comprennent leurs propres centres d'art et grands artistes. La transformation du marché de l'art mondial s'est produite à la suite d'une augmentation énorme de la participation d'artistes des pays asiatiques, notamment la Chine, en réponse au développement économique de ces pays. Les marchés de l'art du Moyen-Orient évoluent dans une direction similaire.

12. New York représente 95\% du marché de l’art des États-Unis (Artprice, 2013: 6). 


\section{RÉSUMÉ}

Le présent article examine la participation des artistes asiatiques au système d'art mondial que dominaient naguère les créateurs et les producteurs européens afin de déterminer si l'hégémonie occidentale est en déclin. Parmi les 500 artistes contemporains avec les montants de ventes aux enchères les plus élevés, $49 \%$ étaient d'origine asiatique en 2011-2012, comparativement à $8 \%$ en 2005. La nationalité des membres des comités de sélection des artistes influe énormément sur la sélection des artistes pour les biennales et les foires artistiques. La nationalité des artistes dont les œuvres sont exposées dans les biennales et vendues aux enchères et dans les galeries dans le cadre de foires artistiques montre qu'il existe une forte «préférence nationale» pour les œuvres produites au pays ou dans la région. Ainsi, le marché de l'art mondial est segmenté en différentes régions, entre l'Orient et l'Occident, chacune avec ses propres artistes et centres d'art d'importance.

Mots clés: marché de l'art, ventes d'art, galeries d'art, biennales, foires artistiques

\section{ABSTRACT}

This article examines participation of Asian artists in the global art system previously dominated by European and American creators and producers in order to assess whether the level of Western hegemony is declining. Among the 500 contemporary artists with the highest levels of auction sales, $49 \%$ came from Asia in 2011-2012, compared to $8 \%$ in 2005 . A major factor in the selection of artists for biennials and art fairs is the nationality of members of the committees that select the artists. National affiliations of artists exhibiting at biennials and being sold at auctions and of galleries participating in art fairs reveal a considerable "home bias": preference for artworks produced locally or regionally. As a result, the global art market is segmented into distinct regions, East and West, each with its major artists and major art centers.

Keywords: art market, art auctions, art galleries, biennials, art fairs

\section{RESUMEN}

Este artículo analiza la participación de los artistas asiáticos en el sistema global de arte, dominado hasta ahora por creadores y productores europeos y estadounidenses, con el fin de evaluar si el nivel de hegemonía occidental se encuentra en declive. En el periodo 2011-2012, de 500 artistas contemporáneos con los más altos niveles de ventas en subastas, $49 \%$ de ellos provenían de Asia, en comparación con el $8 \%$ en 2005 . Un factor importante en la selección de artistas para bienales y ferias de arte es la nacionalidad de los miembros de los comités que seleccionan a los artistas. Las afiliaciones nacionales de los artistas que exponen en las bienales y que venden en subastas y galerías que participan en ferias de arte, revela una considerable "polarización doméstica": una preferencia por obras de arte producidas local y regionalmente. Como resultado, el mercado global de arte se encuentra segmentado en distintas regiones, Este y Oeste, cada una de ellas con sus más importantes artistas y principales centros de arte.

Palabras clave: mercado de arte, subastas de arte, galerías de arte, bienales, ferias de arte 


\section{BIBLIOGRAPHIE}

Adeli, J. (2011), "Translocal art worlds in times of medialization: some observations of India's contemporary art world in transition, » International Quarterly for Asian Studies, vol. 42, n⿳/4 (Novembre), p. 257-277.

«Art Basel Selection Committee for Hong Kong» (2013), www.artbasel.com/en/Hong-Kong/Exhibitingat-the-Show/Selection-Committee

ARTPRICE (2011-2012), The Contemporary Art Market 2011/2012, artprice.com

ARTPRICE (2012), " The emergence of an open and decentralised Asian art market in 2012: contemporary auction turnovers sold in top 20 cities (2011 et 2012)», artprice.com

ARTPRICE (2013a), Contemporary Art Market Annual Report, artprice.com

ARTPRICE (2013b), «The art market in 2012 — the emergence of Asia as a decentralising and liberalising factor in the art market», www.artintheflat.fr/en/le-marche-de-lart/le-marche-de-lart-en-2012

Azımi, R. (2013a), «Chacun veut «sa» biennale», Le Monde, culture\&idées, 7 septembre, p. 2.

Azimi, R. (2013b), «Le marché de l'art n'a pas toujours raison», Le Monde, argent\&patrimoine, 16 octobre, p. 9.

Azımi, R. (2013c), «Les musées français courtisent les collectionneurs chinois», Le Monde, 25 octobre, p. 14.

Azimi, R. et de Changy, F. (2013), "Asia Pacific's art market comes of age», Guardian Weekly, 16 juillet.

Baia Curioni, S. B. et coll. (2015), "Making visible: Artists and Galleries in the Global Art system », dans Velthius, O. et Curioni, S. B. (dir.) Cosmopolitan Canvases: The Globalization of Markets for Contemporary Art, Oxford, Royaume-Uni: Oxford University Press, 55-77.

Barboza, D. et Cox, A. (2013), "Art and fraud in China», International Herald Tribune, 28 octobre, p. 1, 4. Barboza, D. et Bowley, G. (2013), «People», International New York Times, 21 et 22 décembre, p. 21.

Bergman, J. (2013), «Asia's art fair boom: Hong Kong and Singapore compete for cultural top slot», http:// world.time.com/2013/01/21/asias-art-fair=boom-hong-kong-and-singapore-com

Beuve-Méry, A. (2013), "Christie’s à l’assaut de la Chine», Le Monde écodrentreprise, 26 septembre, p. 2. Biennial Foundation (2013), «Biennial map», www.biennialfoundation.org/biennial-map/

Bowley, G. (2013), «Art sales go globe-trotting», International Herald Tribune, 22 août, p. 1, 4.

Bowley, G. et Barboza, D. (2013), «A Chinese auction giant tests the limits of transparency», International New York Times, 16 décembre, p. 6.

Brown, M. (2013), "Qatar's Sheikha Mayassa tops art power list», The Guardian, 24 octobre www. theguardian.com/artanddesign/2013/oct/24/qatar-sheikah-mayassa-tops-art-power-list

Buchrolz, L. (2013), The Global Rules of Art, New York: dissertation de doctorat, Columbia University.

Buchнolz, L. et Wuggenig, U. (2005), «Cultural globalisation between myth and reality: the case of the contemporary visual arts», Glocalogue, $\mathrm{n}^{\circ} 04$.

Burns, A. et Burns, C. (2011), "Qatar revealed as the world's biggest contemporary art buyer», The Art Newspaper, $\mathrm{n}^{\circ} 226$, juillet-août.

Chen, X. (2013), «Hong Kong finds footing in art world», International Herald Tribune, The Art of Collecting, 23 mai, p. I.

«China focus for next Armory fair» (2013), The Art Newspaper, 16 septembre www.theartnewspaper.com/ articles/china-focus-for-next-Armory-fair/30435

"Chinese Contemporary Art Today» (2009), http://web.artprice.com/artmarketinsight/545/ Chinese+Contemporary+Art+today? $1=$ en

Chiu, M. (s.d.), Asian contemporary art: an introduction, www.oxfordartonline.com/public/page/ asiancontintro

Crane, D. (2002), "Culture and globalization: theoretical models and emerging trends », dans Crane, D., Kawashima, N. et Kawasaki, K. (dir.) Global Culture: Media, Arts, Policy and Globalization, New York, Routledge, p. 1-25.

Crane, D. (2014), «Les mondes culturels: des mondes urbains aux mondes globaux», dans Jeanpierre, L. et Rouff, O. (dir.), Intermédiaires et prescripteurs au centre de la création. Une nouvelle sociologie du travail artistique, Paris, Éditions des archives contemporaines, p. 213-236. 
Creative Economy Report (2013), Special edition: widening local development pathways, New York et Paris, UNDP et UNESCO.

DOCUMENTA (13) «Advisors» (2012), http://universes-in-universe.org/eng/bien/documenta/2012/ advisors «Focus: Biennale de Venise, 55e édition» (2013), La Gazette Drouot, ${ }^{\circ} 41$ du vendredi 29 novembre, p. 372-373.

«For Exhibitors/Art Stage Singapore» (2013), www.artstagesingapore.com/exhibitors/

Frazier, D. (2013), "Art Basel comes to Asia, bringing galleries, artists, collectors - and money», www.forbes.com/sites/donaldfrazier/2013/07/22/art-basel-comes-to-asia-bringing-galleries-artistscollectors-and-money

Giddens, A. (1990), The Consequences of Modernity, Oxford: Oxford University Press.

Gioni, M. (2013), «The encyclopedic palace», www.labiennale.org/en/art/exhibition/gioni/

«Gwangju Biennale 2012: curatorial genius or chaos?» (2012), Art Radar Asia, 8 août http://artradarjournal. com/2012/08/08/gwangju-biennale-2012-curatorial-genius-or-chaos

Hamdon, S. (2013), «Dynamic art scene takes wing in Dubai,» New York Times, 20 mars.

HaRris, J. (2013), «Gatekeepers, poachers and pests in the globalized contemporary art system», Third Text, vol. 27, n 4, p. 536-548.

Heilbron, J. (2001), «Échanges culturels transnationaux et mondialisation: quelques réflexions», Regards sociologiques, vol. 22, p. 141-154.

Heinich, N. (2014) Le paradigme de l'art contemporain: Structures d'une révolution artistique, Paris, Éditions Gallimard.

KendzulaK, S. (2013). Top 10 International Fine Art Fairs, About.com Fine Art, http://fineart.about.com/ od/Art-Fairs/tp/Top-10-Fine-Art-Fairs-Antique-Festivals: Around-The-World.htm

Kong, L. (2007), «Cultural icons and urban development in Asia: Economic imperative, national identity, and global city status», Political Geography, vol. 26, p. 383-404.

KRÄUsSL, R. (2015), "Art as an alternative asset class: Risk and return characteristics of the Middle Eastern and Northern African art markets», dans Velthius, O. et Curioni, S. B. (dir.) (2015), Cosmopolitan Canvases: The Globalization of Markets for Contemporary Art, Oxford, Royaume-Uni, Oxford University Press, 147-169.

LAU, J. (2013), «Hong Kong makes it on the world stage», International Herald Tribune, 23 mai, p. 10.

LeChner, F. (2005), «Globalization», dans G. Ritzer (dir.), Encyclopedia of Social Theory, vol. 1 (Thousand Oaks, CA: Sage).

Melikian, S. (2013), "Art market shuts out all but the wealthy», International Herald Tribune, 28 et 29 septembre, p. 25.

Morgner, C. (2014a), «The art fair as network», The Journal of Arts Management, Law and Society, vol. 44, p. 33-46.

Morgner, C. (2014b), «The biennial: The practice of selection in a global art world», Empirical Studies of the Arts, vol. 32, n 2, p. 275-282.

Moulin, R. (2000), Le marché de l'art: mondialisation et nouvelles technologies, Paris, Flammarion.

Pechman, A. (2014), «Next to Burberry, a Monet», International New York Times, 6 juin, p. 12.

Platt, K. H. (2012), «A culture clash with Chinese art», International Herald Tribune, CONTEMPORARY ART: SPECIAL REPORT, 13 juin, p. S4.

Platt, K. H. (2013), «China's Venetian quandary: Chinese artists», International Herald Tribune, THE ART OF COLLECTING: SPECIAL REPORT, 12 juin, p. S2.

Pogrebin, R. (2013), «That deep-pocketed art buyer? It's an oil-rich bidder: Quatar», International Herald Tribune, 23 juillet, p. 1, 7.i

POwER 100 (2013), «A ranked list of the contemporary art world's most powerful figures», http://artreview. com/power_100/

Quemin, A. (2013), «International contemporary art fairs in a "globalized" art market», European Societies, vol. $15, \mathrm{n}^{\circ} 2$, p. $162-177$.

Quemin, A. (2006), «Globalization and mixing in the visual arts: an empirical survey of "high culture" and globalization», International Sociology, vol. 21, $\mathrm{n}^{\circ}$ 4, p. 522-550. 
Quemin, A. (2002), «L'illusion de l'abolition des frontières dans le monde de l'art contemporain international: la place des pays «périphériques» à «l'ère de la globalisation et du métissage»», Sociologie et sociétés, vol. $34, \mathrm{n}^{\circ} 2$, p. 15-40.

Reyburn, S. (2014a), «Speculating on trophy art», International New York Times, 3 mars, p. 10.

Reyburn, S. (2014b), «On the art fair carousel», International New York Times, 31 mars, p. 14.

Robertson, I. (2011), A New Art From Emerging Markets, Farnham, Surrey, UK, Lund Humphries.

Rouet, F. (2013-2), «Les galeries d'art contemporain en France en 2012 », Culture études: économie de la culture et de la communication, 11 juin.

Sagot-Duvauroux, D. (2014), «Galeries: une nouvelle économie», Le Journal des Arts, nº 10, 28 mars au 10 avril, p. 34-35.

VAn Hest, F. (2012), Territorial Factors in a Globalised Art World: The Visibility of Countries in International Contemporary Art Events, Rotterdam, Erasmus Research Centre for Media, Communication and Culture.

Van Hest, F. (2015), «La présence marginale d'œuvres non occidentales sur le marché de l'art contemporain», Revue Proteus-Cahiers des théories de l'art, nº 8, mars, p. 39-55.

Velthuis, O. (2013), «Globalization of markets for contemporary art: why local ties remain dominant in Amsterdam and Berlin», European Societies, vol. 15, n² 2, p. 290-307.

Velthuis, O. et Curioni, S. B. (dir.) (2015), «Making markets global», dans Velthuis, O. et Curioni, S. B. (dir.), Cosmopolitan Canvases: The Globalization of Markets for Contemporary Art, Oxford, RoyaumeUni: Oxford University Press, 1-28.

Vogel, C. (2013), «The gang's all there, talking art in Qatar», International New York Times, 17 octobre, p. 10.

Yogev, T. et Ertug, G. (2015), "Global and local flows in the contemporary art market: the growing prevalence of Asia, dans Velthuis, O. et Curioni, S. B. (dir.), Cosmopolitan Canvases: The Globalization of Markets for Contemporary Art, Oxford, Royaume-Uni: Oxford University Press, 193-212.

«Yuko Hasegawa» (2013), http://universes-in-universe.org/eng/bien/sharjah_biennial/2013/yuo_hasegawa

\section{Sources documentaires}

Foires d'art

Art Basel

Russuth, A. (2013), «Here's the 2013 Art Basel Exhibitor List», http://galleristny.com/2013/02/heres-the=2013-art-basel-exhibitor-list/

Art Basel Hong Kong

https://www.artbasel.com/en/hong-kong

Art Dubaï

"Art Dubai 2013: Participating Galleries ", www.art-agenda.com/shows/art-dubai-2013-participating-galleries/

«Art Stage Singapore: Exhibitors 2013», www.artstagesingapore.com/exhibitors/exhibitors-2013/

Frieze

«Frieze London 2013: Gallery List», http:/friezelondon.com/news/frieze-london-new-design-for-2013/

Biennales

«dOCUMENTA (13) — List of Artists »,

http://universes-in-universe.org/eng/bien/documenta/2012/documenta_13_artists 
Biennale de Gwangju

" $9^{\text {th }}$ Gwangju Biennale: List of Artists»,

http://universes-in-universe.org/eng/bien/gwangju_biennale/2012/artists

Biennale de Sharjah

www.sharjahart.org/biennial/sharjah-biennial-1

Biennale de Venise

http://labiennale.org/en/art/exhibtion/artists/ 